\title{
NGC 2571: An intermediate-age open cluster with a White Dwarf candidate ${ }^{\star}$
}

\author{
E. E. Giorgi ${ }^{1,2}$, R. A. Vàzquez ${ }^{1}$, G. Baume ${ }^{1,3}$, W. Seggewiss ${ }^{4}$, and J.-M. Will ${ }^{4}$ \\ 1 Facultad de Ciencias Astronómicas y Geofísicas, UNLP, and IALP (UNLP-CONICET), \\ Paseo del Bosque s/n, 1900, La Plata, Argentina \\ 2 Fellow of Comisión de Investigaciones Científicas de la Provincia de Buenos Aires, Argentina \\ 3 Post-doctoral fellow of CONICET \\ 4 Sternwarte der Universität Bonn, Auf dem Hügel 71, 53121 Bonn, Germany
}

Received 3 August 2001 / Accepted 18 October 2001

\begin{abstract}
CCD $U B V I$ imaging photometry was carried out in the field of the open cluster NGC 2571. From the analysis of our data we state the cluster is at a distance of $1380 \pm 130 \mathrm{pc}$ and its age is $50 \pm 10 \times 10^{6} \mathrm{yr}$. The cluster mass function has a slope larger than a typical Salpeter's law. There are two notorious features in NGC 2571: the cluster contains a high proportion of stars located below the reference line that are serious candidates to be metallic line stars (probably Am-Fm), and shows also a sharp gap along its main sequence that cannot be explained by a random process nor by a biased rejection of cluster members. A striking blue object was detected in the cluster field that could be a white dwarf candidate.
\end{abstract}

Key words. open clusters and associations: individual: NGC 2571 - stars: luminosity function, mass function H-R diagrams - stars: chemically peculiar - stars: blue stragglers

\section{Introduction}

This article is the first of a series aimed at investigating the spiral structure in the Vela Puppis spur arm through deep $U B V I$ CCD photometry of open clusters.

The open cluster NGC 2571 (C 0816-295, $\alpha_{2000}=$ $\left.8^{\mathrm{h}} 18^{\mathrm{m}} 55^{\mathrm{s}}, \delta_{2000}=-29^{\circ} 36^{\prime} 45^{\prime \prime}\right)$ is located in the direction of the Vela-Puppis spur, at $l=249^{\circ} .1$ and $b=3.5$, in a region of nearly constant interstellar absorption, $A_{V} \approx 0 . \mathrm{m} 3$ from 1 to $4 \mathrm{kpc}$ from the Sun (Vogt \& Moffat 1972; Neckel $\&$ Klare 1980). Three attempts have been made to determine the distance of NGC 2571 using $U B V$ photometry: Lindoff (1968, pg photometry) derives a distance of $2.05 \mathrm{kpc}$ and a colour excess of $E_{(B-V)}=0 .{ }^{\mathrm{m}} 32$. But Clariá (1976, pe) and Kilambi $(1978, \mathrm{pg})$ place the cluster at a distance of $1.25 \mathrm{kpc}$ only under $E_{(B-V)}=0.1$.

On the other hand, Lindoff (1968) and Clariá (1976) agree on the cluster's age, about $30 \times 10^{6} \mathrm{yr}$ based on an absolute magnitude $M_{V}=-1 .{ }^{\mathrm{m}} 9$ of the turn-off point (TO). Kilambi (1978) reduces the TO to $M_{V}=$ -0.5 thus increasing the age to $175 \times 10^{6} \mathrm{yr}$.

As a consequence, subsequent interpreters of the foregoing photometric papers arrived at different conclusions on the nature of the brightest stars of the

Send offprint requests to: E. Giorgi,

e-mail: egiorgi@fcaglp.edu.ar

* Based on observations collected at the European Southern Observatory ESO at La Silla, Chile. cluster: HD 70058 (B3/5) and HD 70078 (B2V), spectral types from Houk (1982). Mermilliod (1981) and Mermilliod \& Maeder (1986) considered them as genuine cluster main-sequence stars and attributed NGC 2571 to their "NGC 3766 age-group" for which they derive the young age of $21 \times 10^{6} \mathrm{yr}$. On the other hand, Kilambi's (1978) faint TO and higher age led Ahumada \& Lapasset (1995) to the conclusion that the two brightest stars must be blue stragglers.

The extensive photographic photometry carried out by Kilambi (1978) shows several interesting features in NGC 2571 which deserve further study: (1) The cluster shows a pronounced gap in the stellar distribution along the main sequence (MS) at $0{ }^{\mathrm{m}} 15 \leq(B-V)_{0} \leq 0^{\mathrm{m}} \cdot 25$. (2) The cluster contains several variable stars that have been identified by comparing photoelectric and photographic photometry. (3) There is an unusual number of stars placed below the reference line in Kilambi's colourcolour diagram, which he attributed to a non-linearity effect in the $(U-B)$ transformations. Unfortunately, the photoelectric photometry performed by Clariá (1976), although more precise, is not deep enough to detect this feature revealed by Kilambi's work.

The present work is therefore aimed to re-examine the reliability of Kilambi's findings with a deeper CCD photometry. In Sect. 2 we describe the observational procedure and the photometric reduction techniques. Section 3 contains the cluster data analysis including a discussion on 


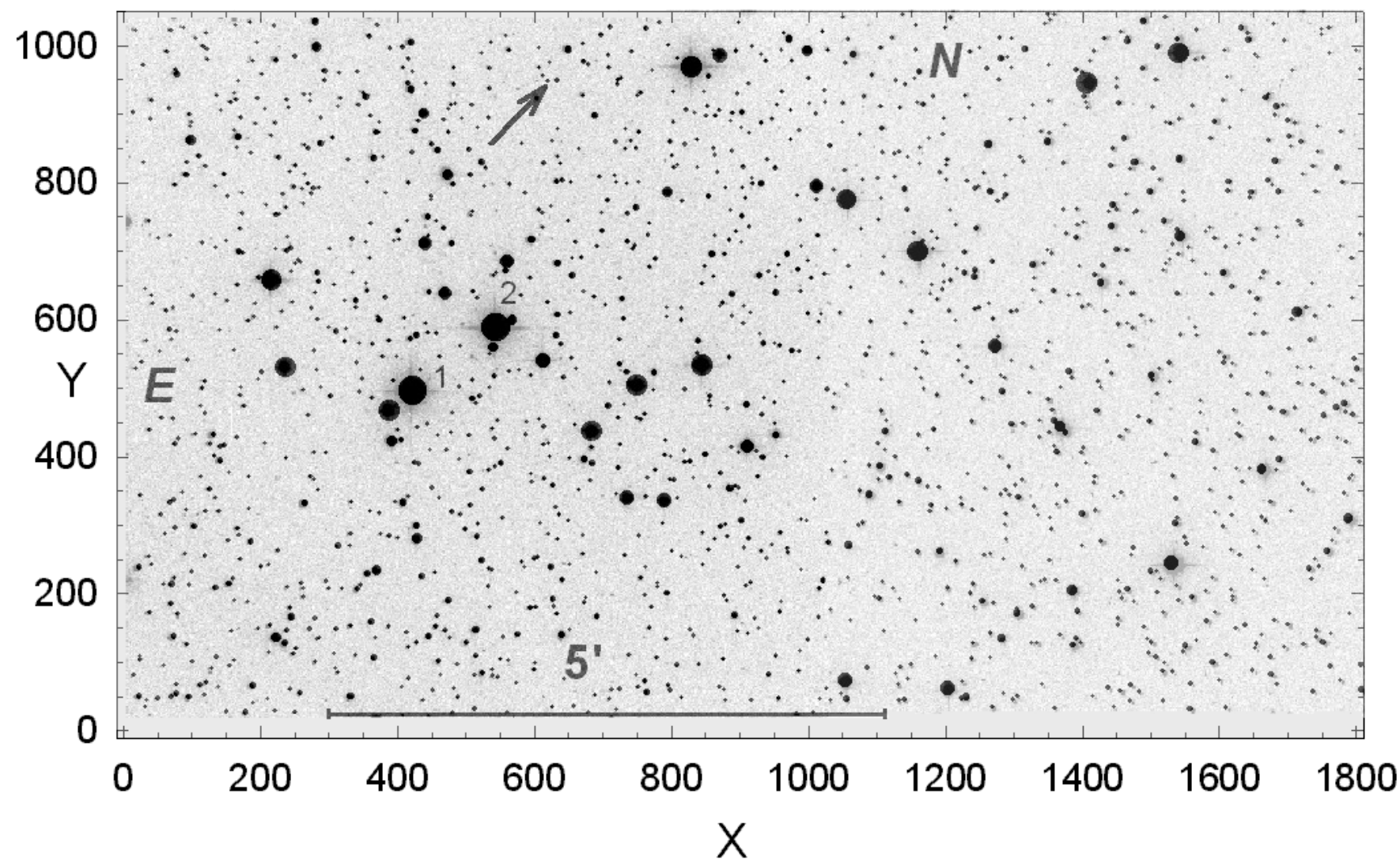

Fig. 1. Finding chart of NGC 2571. North and East are indicated. The arrow gives the position of star No. 1357, a White Dwarf candidate (see Sect. 3.6).

distance and age, on cluster membership, variable stars, peculiar star candidates, and on the main-sequence gap. In addition, we describe briefly the luminosity function (LF) and mass function (MF) of NGC 2571. Section 4 contains our conclusions.

\section{Observations}

We carried out CCD imaging photometry in the KronCousins $U B V I$ system on April 27 and 29, 1994 using a $1024 \times 1024 \mathrm{pxl}$ CCD with a scale of $0.37 / \mathrm{pxl}$ and a field of view of $6.3 \times 6.3$ attached to the 1.54-m Danish telescope at ESO, La Silla, Chile. Figure 1 shows the two overlapping fields that were observed for the cluster NGC 2571. They cover an effective area of $6.3 \times 11^{\prime}$. Whenever possible, the statistics of the faintest stars was maximized taking long frames of same exposure time and combining them. In addition, very short exposures were needed to obtain reliable photometric data for the two brightest stars in the field. However, in this case, it was necessary to apply a shutter timing correction. Details of the exposure times and the number of observations can be found in Table 1. The mean seeing on the entire run was 1.'1 and the quality of both nights was photometric.

Instrumental magnitudes were obtained by point spread function (PSF) fitting using the DAOPHOT package (Stetson 1987) running within IRAF. More than fifteen stars from two standard fields of Landolt (1992) have
Table 1. Journal of science-frame observations and exposure times [s] of NGC 2571. The number of averaged frames is given in parentheses.

\begin{tabular}{l|lllll}
\hline \hline Passband & & $U$ & $B$ & $V$ & $I$ \\
\hline Night 1 & short & 4 & 1 & 1 & 1 \\
& medium & 10 & 10 & 60 & 20 \\
& long & $600(2)$ & $600(4)$ & $300(4)$ & $300(4)$ \\
\hline Night 2 & short & 15 & 3 & 1 & 1 \\
& medium & 300 & 120 & 30 & 20 \\
& long & 900 & $600(4)$ & $300(4)$ & $300(4)$ \\
\hline
\end{tabular}

been observed each night to convert the instrumental magnitudes into the standard system via transformation equations of the following form:

$u=U+u_{1}+u_{2} \times X U+u_{3} \times(U-B)$

$b=B+b_{1}+b_{2} \times X B+b_{3} \times(B-V)$

$v=V+v_{1}+v_{2} \times X V+v_{3} \times(B-V)$

$i=I+i_{1}+i_{2} \times X I+i_{3} \times(V-I)$

where $u_{2}, b_{2}, v_{2}$, and $i_{2}$ are the extinction coefficients, provided by Agnes Metanomski (2000) from Heidelberg, $X U$, $X B, X V$, and $X I$ the air masses for each filter and $u_{1}, b_{1}$, $v_{1}, i_{1}, u_{3}, b_{3}, v_{3}$, and $i_{3}$ the fitting constants. The value of these constants are shown in Table 2.

The final photometric catalogue for 1575 stars is given in self-explanatory format in Table 3 ; it also includes a 
Table 2. Extinction coefficients and fitting constants of the transformation equations.

\begin{tabular}{l|ccc}
\hline \hline & $u_{1}=5.013 \pm 0.016$ & $u_{2}=0.397$ & $u_{3}=-0.000 \pm 0.031$ \\
Night 1 & $b_{1}=2.229 \pm 0.011$ & $b_{2}=0.245$ & $b_{3}=-0.171 \pm 0.011$ \\
& $v_{1}=1.789 \pm 0.035$ & $v_{2}=0.137$ & $v_{3}=-0.138 \pm 0.035$ \\
& $i_{1}=2.664 \pm 0.016$ & $i_{2}=0.052$ & $i_{3}=-0.012 \pm 0.011$ \\
\hline \multirow{3}{*}{ Night 2 } & $u_{1}=5.018 \pm 0.014$ & $u_{2}=0.425$ & $u_{3}=-0.083 \pm 0.023$ \\
& $b_{1}=2.208 \pm 0.009$ & $b_{2}=0.251$ & $b_{3}=-0.173 \pm 0.101$ \\
& $v_{1}=1.675 \pm 0.010$ & $v_{2}=0.142$ & $v_{3}=-0.057 \pm 0.012$ \\
& $i_{1}=2.653 \pm 0.015$ & $i_{2}=0.052$ & $i_{3}=-0.025 \pm 0.013$ \\
\hline
\end{tabular}

cross correlation with other authors and additional information from different sources. The precision of our data is depicted in Fig. 2 that shows the photometric errors from DAOPHOT against the visual magnitude; even at very faint magnitudes the errors of our photometry are quite small. The final errors for stars with $V \leq 18^{\mathrm{m}}$ (including fitting and calibration) are: $\epsilon_{V}=0.023, \epsilon_{B-V}=0.029, \epsilon_{U-B}=0.03, \epsilon_{V-I}=0.04$. The mean values and standard deviations of the internal errors of our photometry, obtained by comparison of stars observed in both nights are: $\epsilon_{V \text { int }}=$ $-0.01 \pm 0.04, \epsilon_{B-V \text { int }}=-0.00 \pm 0.01, \epsilon_{U-B \text { int }}=-0.03 \pm$ $0.10, \epsilon_{V-I \text { int }}=-0.04 \pm 0.05$.

\section{Data analysis}

\subsection{Cluster size}

To estimate the angular diameter of NGC 2571, we computed the surface stellar density using an image of $30^{\prime} \times 30^{\prime}$ retrieved from the Digitized Sky Survey 2-Red (DSS2Red), centred on NGC 2571. The counts were performed in concentric rings around star No. 1 up to $V \approx 18 \mathrm{mag}$ and then divided by their respective surfaces. The final density profile and the respective error bars are depicted in Fig. 3. The surface density decreases with radius up to $\approx 6^{\prime}$ from the cluster centre where it merges into the background stellar density indicated by the dashed line, obtained as the mean of the stellar density outside the apparent boundaries of the cluster. It is quite obvious that the cluster is mostly evident because of its few moderately bright stars instead of having a high stellar density. Within the unavoidable uncertainty we adopt $6^{\prime}$ as the angular radius of NGC 2571 (see also Lindoff 1968). When comparing with the area covered in our survey we conclude that our observations comprise very well the core of the cluster and more than $60 \%$ of its total area. But we note that the bright red star, CD - 295 5941 (= Clariá No. 3) classified as non cluster member by Clariá (1976), is not in our field.

\subsection{Comparison with previous photometric studies and variable stars}

As mentioned above, previous studies of NGC 2571 are reported in the literature. The photometric works of Lindoff,

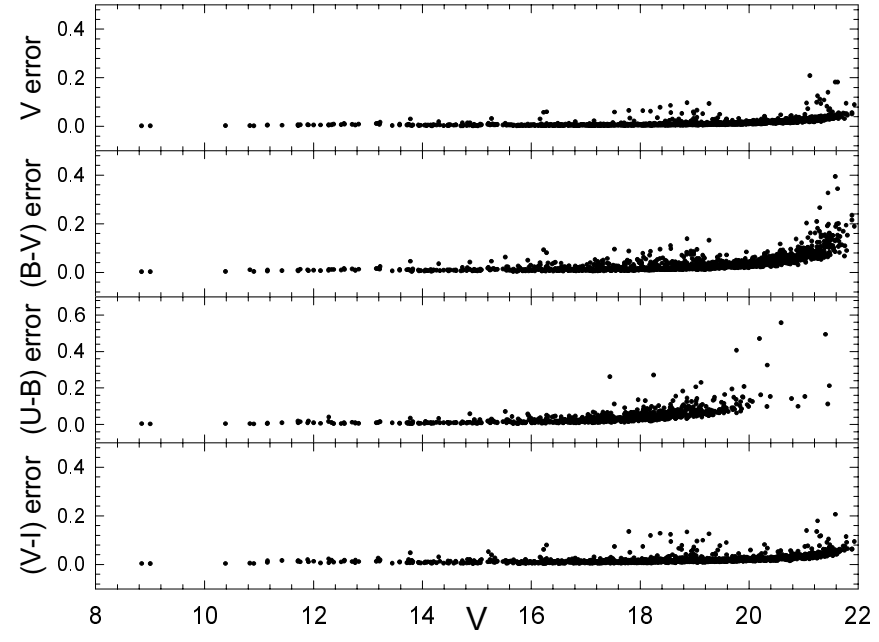

Fig. 2. Photometric errors in colour and magnitude vs. $V$ magnitude as given by DAOPHOT.

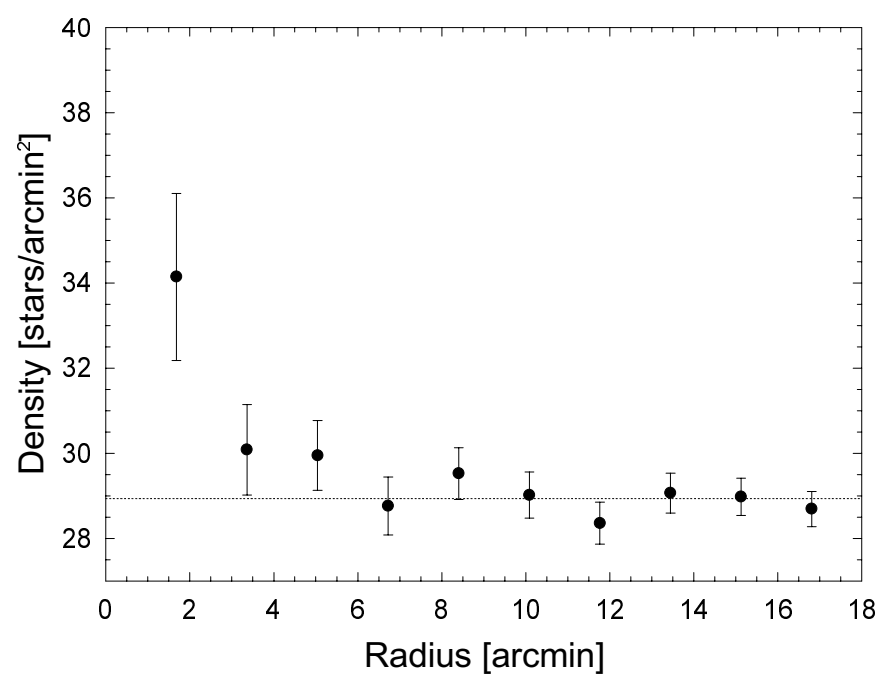

Fig. 3. Density of stars in NGC 2571 as a function of radius. The line is the mean field stellar density.

Clariá and Kilambi provide the most extended data sets to carry out a straight comparison of our photometry with theirs. Table 4 shows the mean differences in $V,(B-V)$, and $(U-B)$ computed in the sense "our values minus others". As it is expected, larger differences are found with the photographic data sets of Lindoff and Kilambi, especially for the $(U-B)$ index. However, what surprises most are the large standard deviations found in $\Delta V$ for all three sets. Even with the more accurate photoelectric photometry of Clariá (1976) the $V$ deviation is so large that we conclude the cluster may contain several variable stars or photometrically unresolved stars. This was first mentioned by Kilambi (1978) who compared his photographic observations to those of Clariá and published a list of variable stars. Here, we search for variable stars by comparing our CCD observations with Clariá's photoelectric photometry. We call a star "variable" if the differences with Clariá's data are larger than 0.1 in magnitude $V$ and in at least one colour. A candidate star is called "probable 
Table 3. Photometric data, spectral types, and coordinates of all stars observed in the open cluster NGC 2571. The complete table with 1575 entries is only available in electronic form at the CDS via anonymous ftp to cdsarc.u-strasbg.fr (130.79.128.5) or via http://cdsweb.u-strasbg.fr/cgi-bin/qcat?J/A+A/381/884.

\begin{tabular}{|c|c|c|c|c|c|c|c|c|c|c|c|c|}
\hline No. & Lind & Clar & Kila & Sp.Type & Ref. & Rem. & $X$ & $Y$ & $V$ & $(B-V)$ & $(U-B)$ & $(V-I)$ \\
\hline 1 & 10 & 1 & 1 & $\mathrm{~B} 2 \mathrm{~V}$ & 1 & & 422.56 & 495.72 & 8.85 & -0.07 & -0.76 & -0.15 \\
\hline 2 & 16 & 2 & 2 & $\mathrm{~B} 3 / 5, \mathrm{~B} 8$ & 1.3 & & 544.73 & 588.43 & 9.00 & -0.09 & -0.67 & -0.11 \\
\hline 3 & 12 & 5 & 5 & B4 & 2 & & 216.93 & 657.26 & 10.38 & -0.07 & -0.28 & 0.00 \\
\hline 4 & 24 & 7 & 7 & B5 & 2 & & 845.34 & 532.86 & 10.83 & -0.02 & -0.39 & -0.13 \\
\hline 5 & 75 & 6 & 6 & K0 & 2 & var? & 829.68 & 968.29 & 10.90 & 1.12 & 1.29 & 1.36 \\
\hline 6 & & 8 & 8 & & & & 1540.64 & 988.93 & 11.15 & 0.04 & -0.02 & 0.02 \\
\hline 7 & 68 & 9 & 9 & B5 & 2 & & 1055.79 & 774.39 & 11.16 & -0.01 & -0.24 & 0.00 \\
\hline 8 & 25 & 13 & 13 & B5 & 2 & & 750.39 & 504.01 & 11.42 & 0.04 & -0.21 & -0.10 \\
\hline 9 & 11 & 16 & 16 & B8 & 2 & & 237.46 & 530.24 & 11.72 & 0.01 & -0.08 & -0.08 \\
\hline 10 & 18 & 17 & 17 & $\mathrm{~A} 0$ & 2 & & 683.93 & 436.88 & 11.72 & 0.13 & 0.09 & 0.05 \\
\hline 11 & & 19 & 19 & & & & 1160.40 & 698.73 & 11.76 & 1.04 & 0.66 & 1.03 \\
\hline 12 & & 23 & 23 & & & & 1407.14 & 945.06 & 11.90 & 0.07 & 0.00 & 0.05 \\
\hline 13 & & 22 & 22 & B6 & 2 & & 388.32 & 466.69 & 11.91 & 0.04 & -0.08 & -0.03 \\
\hline 14 & & 25 & 25 & & & & 560.67 & 684.72 & 12.00 & 0.04 & 0.08 & 0.01 \\
\hline 15 & 19 & 26 & 26 & & & & 736.00 & 338.99 & 12.13 & 0.06 & 0.04 & 0.02 \\
\hline 16 & & 27 & 27 & B9 & 2 & & 1203.26 & 61.27 & 12.28 & 0.11 & 0.16 & 0.12 \\
\hline 17 & 148 & & 110 & & & & 1529.70 & 244.12 & 12.29 & 1.68 & 1.87 & 1.68 \\
\hline 18 & & 28 & 28 & B9 & 2 & & 1272.94 & 561.11 & 12.33 & 0.13 & 0.14 & 0.11 \\
\hline 19 & 20 & 29 & 29 & A1 & 2 & & 790.28 & 335.75 & 12.37 & 0.09 & 0.16 & 0.07 \\
\hline 20 & 14 & 32 & 32 & & & & 470.12 & 637.68 & 12.51 & 0.29 & 0.19 & 0.38 \\
\hline 21 & 69 & 33 & 33 & $\mathrm{~A} 0$ & 2 & pec, var & 1012.53 & 793.81 & 12.56 & 0.08 & 0.23 & 0.16 \\
\hline 22 & 22 & 31 & 31 & & & var & 911.28 & 414.62 & 12.57 & 1.05 & 0.89 & 1.19 \\
\hline 23 & 74 & 36 & 36 & & & pec, var & 871.26 & 985.17 & 12.77 & 0.11 & 0.26 & 0.22 \\
\hline
\end{tabular}

variable" if it shows differences larger than $0.1 \mathrm{mag}$ in $V$ or one colour alone. Therefore, we state that star No. 5 is a "probable variable" and stars Nos. 21, 22, 24, 27, 30, 33, and 38 are "variable stars". Table 3 includes details on variability in NGC 2571. Just when this article was submitted, Moitinho (2001) kindly provided us with his unpublished data on NGC 2571 for comparison purposes (as shown in Table 4). Although there is a very good agreement of both CCD data sets, some of our variable stars were found to show $V$ and colour differences.

\subsection{Cluster memberships}

Some astrometric data for NGC 2571 stars can be found in the Hipparcos and Tycho Catalogues (ESA 1997): proper motions are available for ten stars, and four stars also have parallax measures (see also Baumgardt et al. 2000). Unfortunately, these data have such large errors that they cannot be used to derive membership probability and cluster distance. Therefore, we have to rely on photometric data alone.

There is some discussion in the literature on the question of membership in star clusters. Abt (1979) claims that proper motions constitute the basic criterion for reliable cluster membership. However, as stated by Turner (1993) and emphasized by Forbes (1996), photometric membership is good enough when it relies on a careful inspection of the two-colour (TCD) and colour-magnitude diagram (CMD) and on a consistent reddening solution.
Figures 4-6 show the TCD and the CMDs of stars in the field of NGC 2571 where the bluest stars are fitted to the intrinsic line for stars of luminosity class $\mathrm{V}$ from Schmidt-Kaler (1982) shifted by $E_{(B-V)}=0.1$. The diagrams indicate that NGC 2571 emerges from a star field mainly composed of old disk population stars. For membership assessment each star was checked for a consistent position in both diagrams. This is easily done for stars down to $V=15.5$ and $(B-V)=0{ }^{\mathrm{m}} 3$; but it would be unrealistic longward of this limit because the fainter the star, the larger the field star contamination. Thus, after having identified the brightest cluster members (large filled circles in Figs. 4-6) we carried out a provisional ZAMS fitting. Then, we included as additional members those stars located up to $\approx 1$ mag above the ZAMS having $(B-V)$ and $(U-B)$ indices with consistent positions in the TCD of Fig. 4. This procedure allowed us to include stars down to approximately $V=17^{\mathrm{m}} 5$ (open circles in Figs. 4-6).

Spectral classification, as indicated in Table 3 , is scarce. Nevertheless, the MK types for the brightest stars Nos. 1 and 2, B2 V and B3/5 (Houk 1982), support the cluster membership of these stars.

To get the intrinsic colours of the cluster members we applied the well-known relations $E_{(U-B)} / E_{(B-V)}=$ $0.72+0.05 E_{(B-V)}$ and $(U-B)_{0}=3.69(B-V)_{0}+0.03$ (Golay 1974; Feinstein et al. 1986) to the eleven bluest members, with $(B-V)<0.20$, that have a unique reddening solution. As an average we get $E_{(B-V)}=0.10 \pm$ 0.04 (s.d.) and $E_{(U-B)}=0.07 \pm 0.03$ (s.d.) in complete agreement with previous values found by Clariá (1976) 
Table 4. Differences of mean magnitudes and of colours with previous studies. $N$ is the number of stars in common.

\begin{tabular}{lcrrrr}
\hline \hline Author & $\Delta(V)$ & $\Delta(B-V)$ & $\Delta(U-B)$ & $\Delta(V-I)$ & $N$ \\
\hline Lindoff (1968) & $0.05 \pm 0.16$ & $0.01 \pm 0.18$ & $0.20 \pm 0.45$ & 51 \\
Clariá (1976) & $0.04 \pm 0.11$ & $-0.04 \pm 0.07$ & $0.04 \pm 0.07$ & & 30 \\
Kilambi (1978) & $0.11 \pm 0.12$ & $-0.03 \pm 0.13$ & $-0.01 \pm 0.14$ & & 44 \\
Moitinho (2001) & $0.00 \pm 0.06$ & $0.00 \pm 0.07$ & $0.03 \pm 0.14$ & $-0.03 \pm 0.06$ & 919 \\
\hline
\end{tabular}

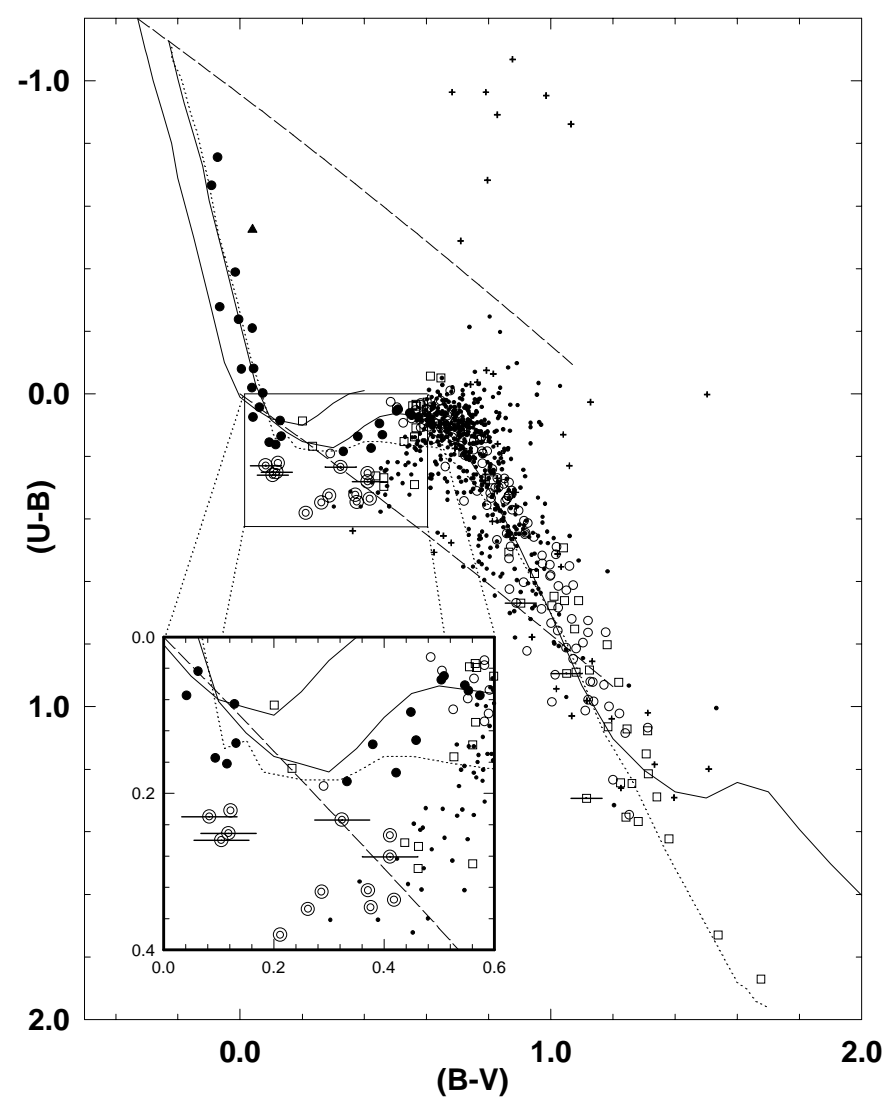

Fig. 4. Two-colour diagram (TCD) $[(U-B)$ vs. $(B-V)]$ of stars in the field of NGC 2571. Symbols are: • bright cluster members; $\circ$ fainter cluster members up to 1 mag above the ZAMS; $\square$ non-members; · distant field stars; + stars with large photometric errors; double circles: probable metallic line stars; symbols with horizontal bar: variable stars; black triangle: probable white-dwarf. - The relation for luminosity class V from Schmidt-Kaler (1982) is shown twice (solid lines): first, unshifted (in the upper left part), and second, shifted by $E_{(B-V)}=0.1$ along the standard reddening path (dashed lines). The dotted line is the relation for giant stars. - Insert: magnification of the lower left part of the TCD.

and Kilambi (1978), respectively. These mean values were used to correct the colours of the rest of the cluster members.

Concerning the reddening law towards NGC 2571, we show in Fig. 7 the TCD $[(B-V)$ vs. $(V-I)]$ with the intrinsic lines of stars of luminosity classes $\mathrm{V}$ and III from Cousins (1978) and the path of normal reddening $E_{(V-I)} / E_{(B-V)}=1.244$ from Dean et al. (1978). The coincidence between the bulk of our data with this

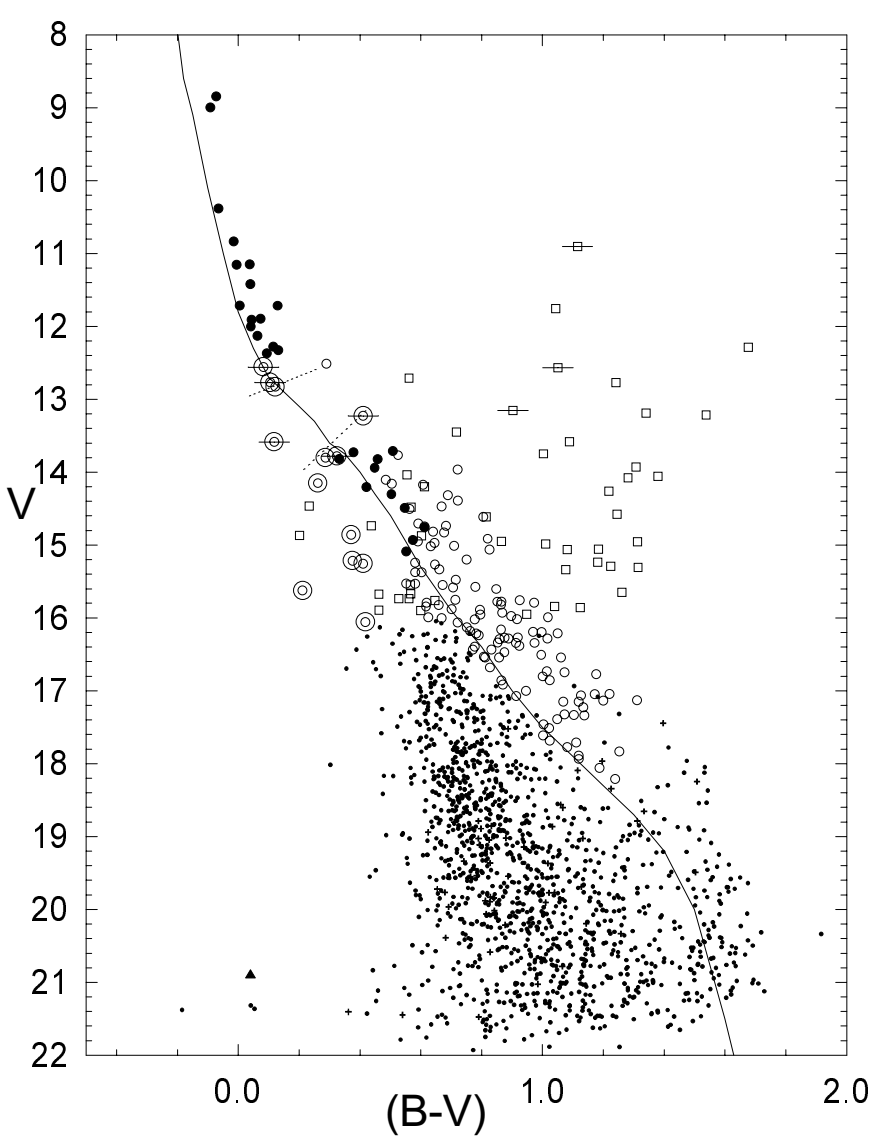

Fig. 5. Colour-magnitude diagram (CMD) $[V$ vs. $(B-V)]$ of stars in the field of NGC 2571. Symbols as in Fig. 4. The solid line represents the Schmidt-Kaler (1982) ZAMS. The dotted lines show the position of the gap (see Sect. 3.7).

reddening line suggests that $R=3.1$ holds in this region. Knowing now that the reddening law is normal in this site, we decided to calculate the absorption-corrected magnitudes through the standard expression $V_{0}=V-$ $3.1 E_{(B-V)}$.

\subsection{Distance and age}

To derive the distance modulus of NGC 2571 we fitted the Schmidt-Kaler (1982) ZAMS to the de-reddened MS. The best fits in both, the $V_{0}$ vs. $(B-V)_{0}$ and $V_{0}$ vs. $(U-B)_{0}$ planes, were achieved for a distance modulus of $V_{0}-M_{V}=$ $10.7 \pm 0.2 \mathrm{mag}$ (error from best-eye inspection), corresponding to a distance of $d=1380 \pm 130$ pc. Our distance is thus larger than the previous values derived by Clariá and Kilambi (10. 49 and 10. 42 , respectively). 


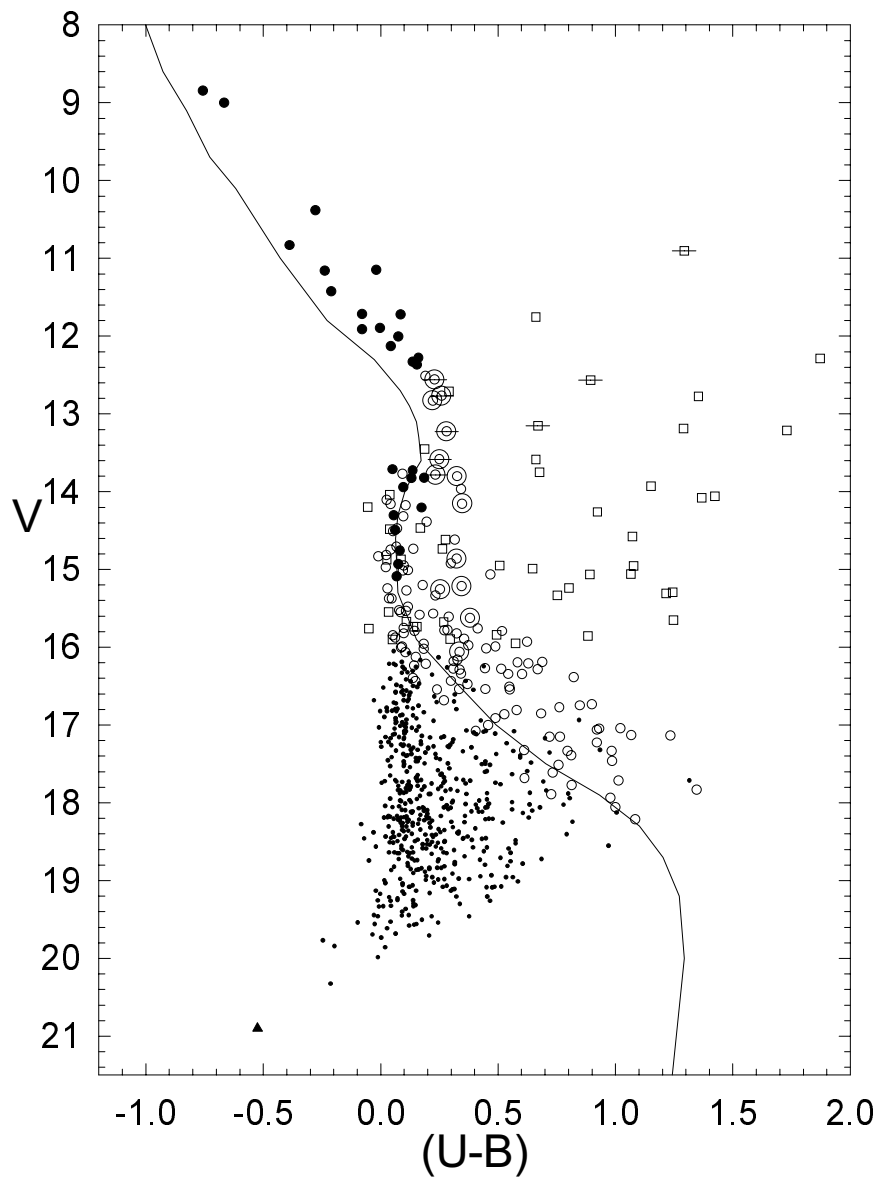

Fig. 6. CMD $[V$ vs. $(U-B)]$ of stars in the field of NGC 2571. Symbols as in Fig. 4. The solid line represents the SchmidtKaler (1982) ZAMS.

This is probably caused by the fact that we fitted the ZAMS to the lower envelope of stars in the corrected CMDs.

The age of NGC 2571 was estimated through a set of isochrones obtained from evolutionary models of Girardi et al. (2000) which were computed with mass loss and overshooting. Figure 8 shows the isochrones superimposed on the corrected CMD (a similar age is obtained when using Schaller et al.'s (1992) isochrones). The two isochrones of 39 and $63 \mathrm{Myr}$, respectively, permit the best fit of the upper part of NGC 2571. Another age estimation can be obtained from the relation between the observed position of the blue TO and $\log ($ age $)$ as given by Meynet et al. (1993). With the TO at $(B-V)_{0}=-0 .{ }^{\mathrm{m}} 15$, NGC 2571 is 80 Myr old. Since the location of the TO is highly uncertain (as it depends on the way the ZAMS is fitted) and for the sake of coherence with our previous works, we prefer an average age obtained through isochrone fitting. Therefore, we adopt $50 \mathrm{Myr}$ as the mean age estimate for NGC 2571. This age disagrees with previous values of 27 and 175 Myr given by Clariá and Kilambi, respectively. Ahumada \& Lapasset (1995) suggest that the two brightest stars are blue stragglers, as they are located far above the cluster TO if an age of $175 \mathrm{Myr}$ is adopted. With the

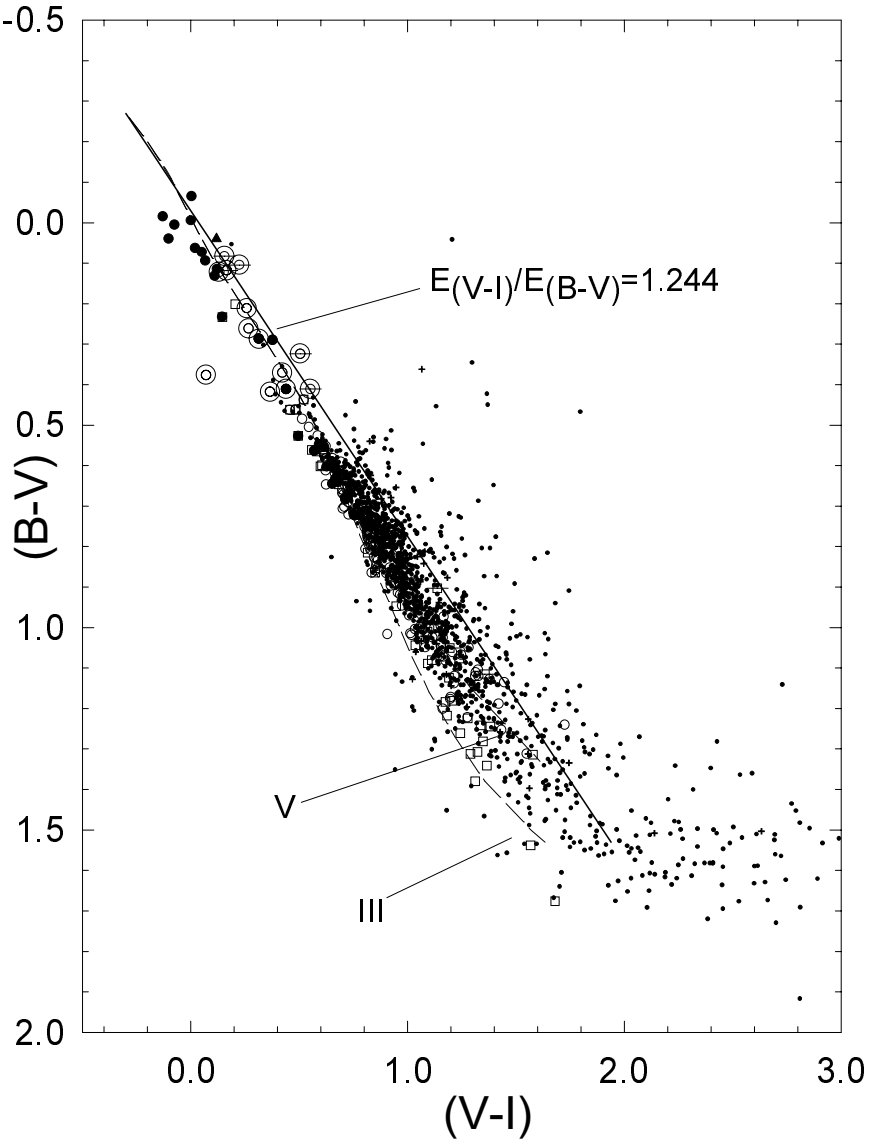

Fig. 7. TCD $[(B-V)$ vs. $(V-I)]$ of stars in the field of NGC 2571. The dashed lines represents the intrinsic location of stars of luminosity classes V and III from Cousins (1978). The solid line represent the reddening line $E_{(V-I)}=1.244 \cdot E_{(B-V)}$, corresponding to $R=3.1$. Symbols as in Fig. 4 .

age obtained here (and, of course, also with Clariá's value) these two stars are not blue stragglers.

\subsection{Peculiar stars in NGC 2571?}

The TCD of NGC 2571 displays a striking feature: 13 stars are situated below the reference line at $0 \leq(B-V) \leq 0 \mathrm{~m} 5$ (Fig. 4) and, simultaneously, fall below the reddening line of an A0V-type star as shown in the insert of Fig. 4. Such a location would indicate that they are A/F-type stars with anomalous colours. The same feature is noticed in Kilambi's (1978) Fig. 2.

Feinstein (1974) demonstrated with a sample of 103 stars of type Am and Ap that the TCD $[(U-V)$ vs. $(V-I)]$ segregates very well the normal from peculiar stars of this kind (see his Fig. 3). This is exactly what we see in our Fig. 9, where these "peculiar stars" show, in general, quite different locations compared to the supposedly normal stars. One is tempted to conclude that these stars are metallic line stars, namely Am stars.

In fact, the Am phenomenon (i.e. strong metallic lines but extremely weak calcium lines) is explained by the radiative diffusion theory and makes a star lie below the MS 


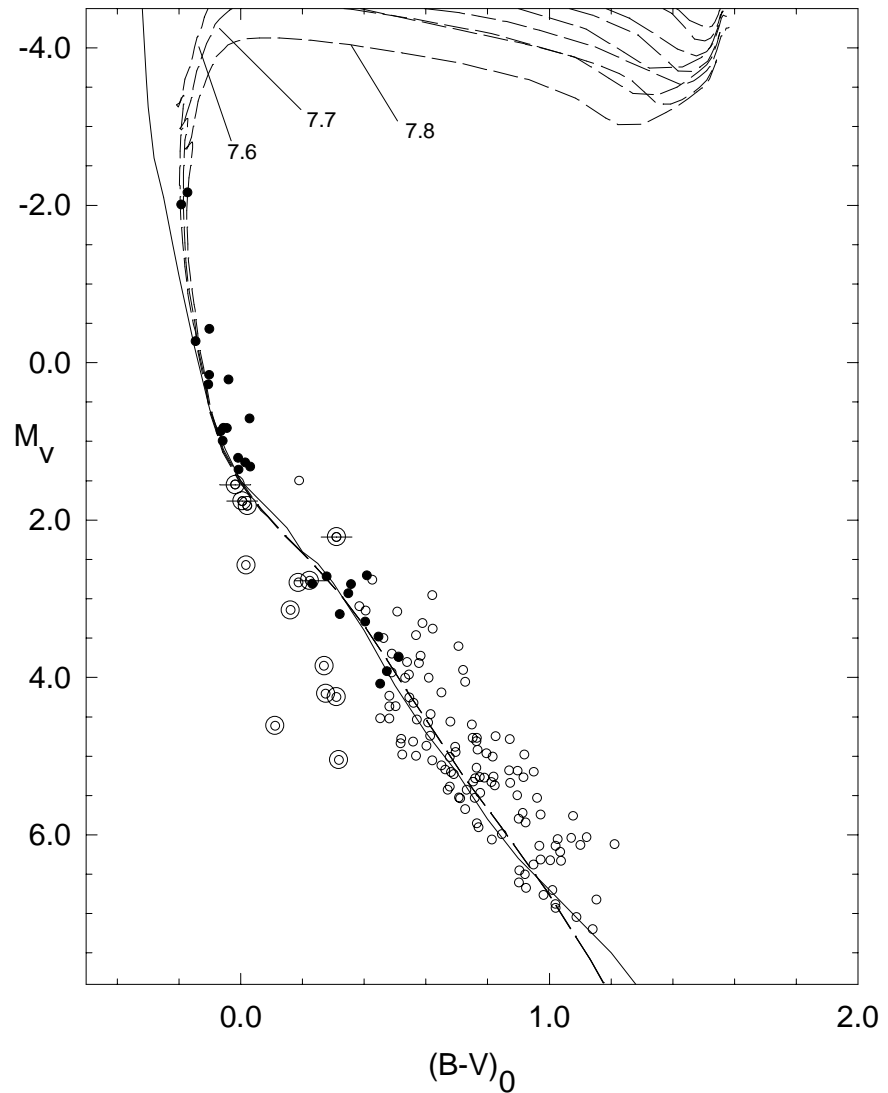

Fig. 8. CMD $\left[M_{V}\right.$ vs. $\left.(B-V)_{0}\right]$ of NGC 2571 . The solid line is the Schmidt-Kaler (1982) ZAMS fitted to a corrected distance modulus of 10.7 . Dashed lines are isochrones from Girardi et al. (2000); numbers indicate $\log ($ age). Note the gap on the main-sequence and the probable metallic-line stars (double circles).

in the TCD because the metallic lines block the star's continuum radiation, especially in the $U$ band. Besides, most of the Am stars ( $\approx 95 \%$ ) belong to binary systems (Berthet 1992), so that light variations could be expected among our "candidates". In particular five of them are variable and three of them lie on or above the ZAMS in the CMD (Fig. 5). One should be cautious in these cases as the Delta Scuti stars are also variables of spectral types AF but more evolved than Am stars (Eggen 1976; Breger 1979).

Am/Ap stars are also frequent members of open cluster and associations with ages $10^{6}-10^{8}$ yr (see Abt 1979 and references therein). If our Am candidates are cluster members their absolute magnitudes go from +1.5 down to $+5 \mathrm{mag}$, as shown in Fig. 8, becoming thus fainter than the lower limit given by Abt (1979): $\approx+3$ mag. This would be a valid argument to reject the faintest of them as cluster members. However, even these faintest stars still could be late Am, viz. Fm-type, stars, if we really see a descending sequence of metallic line stars belonging to the cluster. Malaroda (1973) lists metallic line stars as late as F8. That would explain the faintness of some of our candidates. Unfortunately, there is no way, photometrically

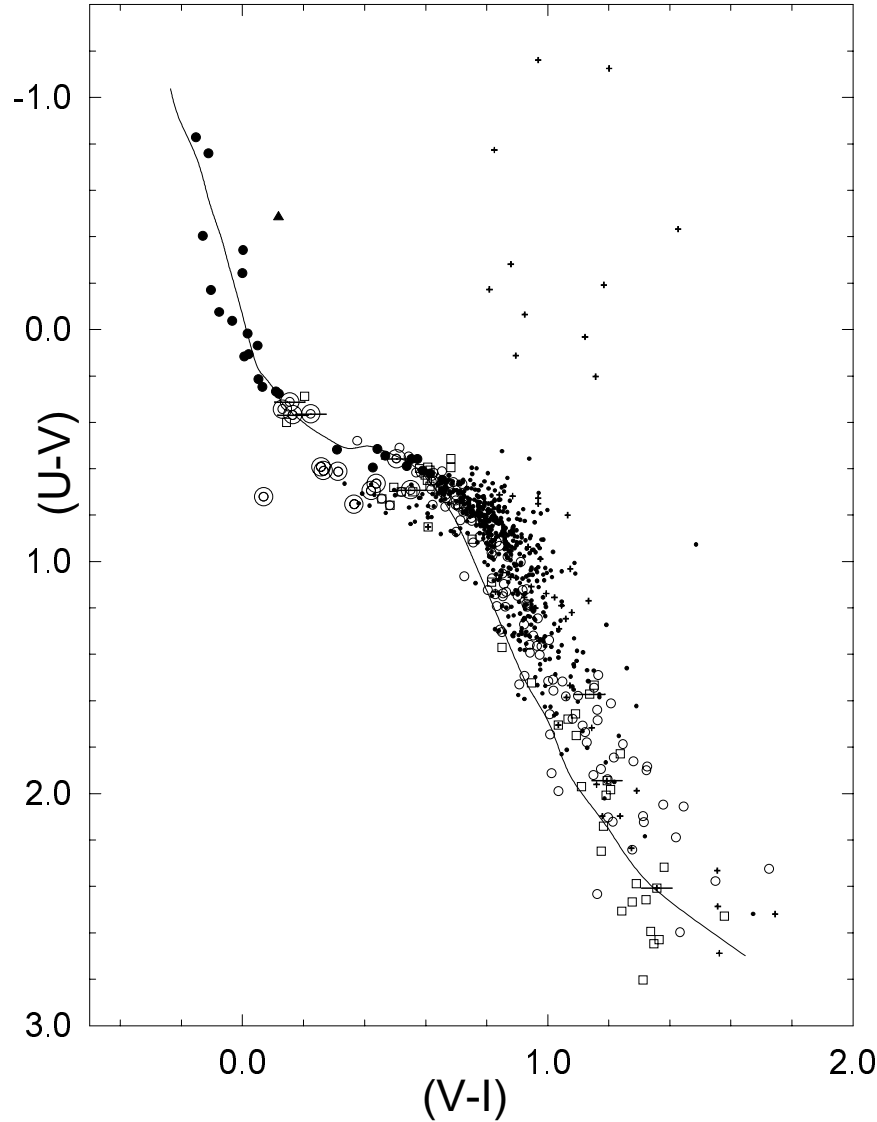

Fig. 9. TCD $[(U-V)$ vs. $(V-I)]$ of NGC 2571. Symbols as in Fig. 4. The intrinsic location of stars of luminosity class $\mathrm{V}$ is indicated.

speaking, to address this subject; spectroscopy is clearly needed.

\subsection{A White-Dwarf star?}

The filled triangle in Fig. 4 shows a blue star (No. 1357 indicated by the arrow in Fig. 1) which is very faint as seen in Figs. 5 and $6(V=20.9)$. The position of this object in the diagrams led us to think of it as a White Dwarf (WD) candidate. The discovery of WDs in the 70 Myr clusters, Pleiades and NGC 2422 (Luyten \& Herbig 1960), allows us to speculate that WD can descend from intermediate massive stars (Romanishin \& Angel 1980). Using Bergeron et al.'s (1995) tables that provide relations between absolute magnitudes, colour indices, temperatures and cooling ages for pure helium and pure hydrogen WDs [log $g=8]$, we shall derive the parameters of star No. 1357. If our candidate were a cluster member and if we account for the fitting error in the distance modulus of NGC 2571 and the mean cluster excess $E_{(B-V)}$, its absolute magnitude would be $M_{V}=9.89 \pm 0.2$. Such an absolute magnitude prevents this star from being a pure helium WD member because it should be fainter. It is, however, completely concordant with a pure hydrogen WD member having a temperature of $\approx 30600 \pm 2000 \mathrm{~K}$, proper for a "young" WD with a 
cooling age from $11_{-3}^{+10}$ Myr. If instead of using the star magnitude we use the $(B-V)$ colour index (including its error), Bergeron et al.'s Table 3 gives a lower temperature, of $21000 \pm 3000 \mathrm{~K}$, and accordingly a larger cooling age of $74_{-30}^{+50} \mathrm{Myr}$, for a pure hydrogen WD.

If star No. 1357 is a field WD we can still estimate its distance and temperature with Bergeron et al.'s tables. We first derive the temperature from the observed $(B-V)$ colour (assuming no reddening or a maximum reddening of $E(B-V)=0.1)$ and then the corresponding WD's absolute magnitude $M_{V}$. We found that if star No. 1357 is a pure hydrogen WD, its distance would range from 800 to $1000 \mathrm{pc}$ and its temperature from 15000 to $23000 \mathrm{~K}$; if it is a pure helium WD, the distance would range from 600 to $900 \mathrm{pc}$ with temperature values from 10000 to $20000 \mathrm{~K}$.

\subsection{The gap}

As already noticed by Kilambi (1978), there is a notorious gap along the MS of NGC 2571 centred at $M_{V}=2$. 1 and $(B-V)_{0}=0.17$ with sizes of 1.2 and 0.25 , respectively (see Figs. 5 and 8 ). Our photometry comprises many more stars than any previous work in this area of the CMD so that no incompleteness effect can be invoked to explain the gap. The literature reports the existence of gaps in several open clusters:

- Mermilliod (1976) found "hot" gaps between spectral types B7 and B8 $\left((B-V)_{0} \sim-0.10\right)$ in young open clusters having ages lesser than the Pleiades;

- Phelps \& Janes (1993) found this feature in Berkeley 7 in the mass range $3 \ldots 4 \mathcal{M}_{\odot}$, in NGC $637\left(8 \ldots 12 \mathcal{M}_{\odot}\right)$, and in Berkeley $62\left(5 \ldots 8 \mathcal{M}_{\odot}\right)$;

- Forbes (1996) found a gap between $0 \leq(B-V)_{0} \leq 0$ m. 20 in NGC 6531;

- A gap (not so obvious) can be observed, too, in NGC 3293 (Herbst \& Miller 1982);

- High-precision astrometry and photometry of the Hyades reveal two gaps along the clusters MS (de Bruijne et al. 2000);

- A detailed description of gaps in several other open clusters has recently been given by Rachford \& Canterna (2000).

The attempts to explain the gaps found in open clusters range from the onset of convection in the stellar envelopes (Böhm-Vitense \& Canterna 1974) to peculiarities in the Balmer jump and Balmer lines as suggested by Mermilliod (1976). Ulrich (1971a, 1971b) has proposed that gaps in some open cluster might be produced by the presence of ${ }^{3} \mathrm{He}$ isotopes that halt the gravitational contraction $1 \ldots 2$ mag above the MS. While Mermilliod's gaps occur in the range of B-type stars, Böhm-Vitense \& Canterna's (and also Ulrich's) gaps are found at less massive stars.

Apparently, the NGC 2571 gap is not produced by a biased estimate of membership: the gap is evident in Fig. 5 even without subtracting any star so that a wrong rejection of members in our analysis is unlikely.
Following Scalo's (1986) discussion of the statistical nature of the initial mass function (IMF), we can compute the probability of finding a gap in the MS: if the cluster has a continuous mass distribution from small masses up to a mass $m_{1}$, no stars from $m_{1}$ to $m_{2}$, and $N$ stars more massive than $m_{2}$, then the probability that this lack of stars is caused by a random process is

$P_{\text {gap }}=\left(m_{2} / m_{1}\right)^{(-N \times x)}$

(where $x$ is the slope of the MF), if the masses of the consecutively formed stars are independent of each other. With our data, computing (adopting in advance the findings of Sect. 3.8) the probability of a gap between $m_{1}=1.6 \mathcal{M}_{\odot}$ and $m_{2}=2.2 \mathcal{M}_{\odot}(N=19$ and $x=1.74)$ is $P_{\text {gap }} \approx 2 \times 10^{-5}$, a value that is difficult to explain by a random process. That the gap in NGC 2571 was caused by a peculiar mass segregation process would be rather improbable too: Kilambi's photometry covers a cluster area larger than ours probably including coronal members (if they exist) and the gap remains stable in his observations. Therefore, we conclude that the gap of NGC 2571 is real. We note that the position of the gap in NGC 2571 is a bit bluer than $(B-V)_{0}=0.25$ as quoted by Böhm-Vitense \& Canterna (1974) and Böhm-Vitense (1982).

\subsection{Luminosity and mass function of NGC 2571}

The luminosity function LF of a star sample is defined by

$\mathrm{d} \log \left(N\left(M_{V}\right)\right) / \mathrm{d} M_{V}=\gamma M_{V}$,

where $\operatorname{d} \log \left(N\left(M_{V}\right)\right) / \mathrm{d} M_{V}$ is the logarithm of the number of stars having absolute magnitudes $M_{V} \pm \mathrm{d} M_{V} / 2$. The star counts of NGC 2571 members in bins of $\Delta M_{V}=1 \mathrm{mag}$ size are given in Table 5 and illustrated in Fig. 10. The slope $\gamma$ of the LF was determined by an unweighted fit through the points ignoring the two lowest bins which are supposed to be affected by incompleteness. The fit yields $\gamma=0.19 \pm 0.02$. Note that the bin $+2 \cdots+3$ clearly shows the effect of the gap.

Figure 10 also shows for comparison the luminosity function given by Kilambi (1978). Because our cluster distance is slightly larger than that found by Kilambi, the two brightest stars are less bright in his histogram than in ours. No noticeable differences can be seen except that our photometry allows us to define very well the LF down to dwarf stars of spectral-type G and K.

The cluster's MF describes the distribution of stars over mass intervals. In particular, if such a description is referred to the moment of the cluster formation, it is called the initial mass function IMF. NGC 2571 is an intermediate-age cluster and, as a consequence, there is a possibility that evolutionary effects have introduced some distortion in its star distribution due to the loss of massive stars. Assuming a power law (see e.g. Scalo 1986), the slope of the MF is given by

$x=-\log (\mathrm{d} N / \mathrm{d}(\log \mathcal{M})) / \log \mathcal{M}$, 
Table 5. LF and MF of NGC 2571. $N$ is the number of stars per bin.

\begin{tabular}{rr||rrrc}
\hline \multicolumn{2}{c||}{ LF } & \multicolumn{5}{c}{$\mathrm{MF}$} \\
\hline$M_{V}$ bins & \multicolumn{1}{c||}{$N$} & $\overline{\mathcal{M}}$ & $\log \overline{\mathcal{M}}$ & $\mathrm{d} N$ & $\log \left(\frac{\mathrm{d} N}{\mathrm{~d}(\log \overline{\mathcal{M}})}\right)$ \\
\hline$-3 \cdots-2$ & 2 & & & & \\
$-2 \cdots-1$ & 0 & & & & \\
$-1 \cdots 0$ & 2 & 7.85 & 0.90 & 2 & 0.89 \\
$0 \cdots 1$ & 8 & 3.41 & 0.53 & 4 & 1.26 \\
$1 \cdots 2$ & 8 & 2.33 & 0.37 & 13 & 1.87 \\
$2 \cdots 3$ & 8 & 1.40 & 0.15 & 62 & 2.31 \\
$3 \cdots 4$ & 21 & 0.86 & -0.06 & 61 & 2.31 \\
$4 \cdots 5$ & 33 & & & & \\
$5 \cdots 6$ & 38 & & & & \\
$6 \cdots 7$ & 22 & & & & \\
$7 \cdots 8$ & 1 & & & & \\
\hline
\end{tabular}

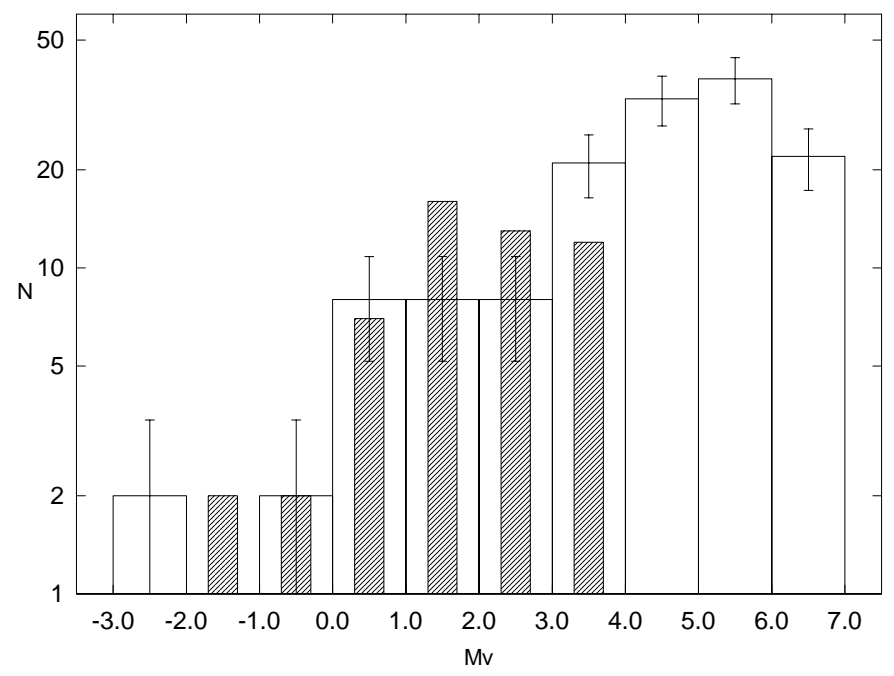

Fig. 10. The LF of NGC 2571 constructed with likely and probably members (cleaned columns). Kilambi's (1978) data are superimposed (shaded columns). Errors bars are indicated.

where $\mathrm{d} N / \mathrm{d}(\log \mathcal{M})$ is the number of stars within the mass intervall $\mathcal{M} \pm \mathrm{d} \mathcal{M} / 2$.

To estimate the MF, we first transformed the corrected data of the member stars from the $\left(M_{V}\right.$ vs. $\left.(B-V)_{0}\right)$ plane to the $\left(\log L / L_{\odot}\right.$ vs. $\left.\log T_{\text {eff }}\right)$ plane, where the bolometric corrections were taken from Schmidt-Kaler (1982). The ZAMS's masses of each stars are obtained using an interpolation procedure between the two closest evolutionary tracks of Schaller et al. (1992). The two brightest stars, having spectroscopic classification, were transformed using the spectral information instead of their colour indices. Because of the gap, once all the stellar masses were obtained, we scaled bin sizes arbitrarily to avoid empty bins. As cluster members go down to $V=17^{\mathrm{m}} 5$ while our magnitude limit is $\approx 22^{\mathrm{m}}$, we are relatively free from incompleteness effects in the low mass bin. By fitting the five mass points of Table 5 using an un-weighted least squares method, we obtain $x=1.66 \pm 0.28$ but if we neglect the most massive bin, the fitting yields $x=1.74 \pm 0.55$, representing thus the MF of the cluster MS stars. These two values are larger than the typical slope of $x=1.35$ given by Salpeter (1955) for field stars. Tarrab (1982) placed this cluster in the age group of NGC $3766\left(2.21 \times 10^{7} \mathrm{yrs}\right)$ whose mean slope is $x=1.58$. The slope she assigned to NGC 2571, with $x=2.30 \pm 0.65$ even larger than ours, was obtained using only 20 stars in the mass range 2.2 to $10 \mathcal{M}_{\odot}$ which is unrealistic in view of the mass values and star numbers given in Table 5 . The actual age of NGC 2571 indicates that the cluster must be placed in Tarrab's (1982) age group of the $\alpha$ Persei cluster $\left(5.13 \times 10^{7} \mathrm{yrs}\right)$ characterized by a mean slope of $x=1.65$.

\section{Conclusions}

- NGC 2571 is a moderate young open cluster of $50 \mathrm{Myr}$, located in the association Vela-Puppis at a distance of $1380 \pm 130 \mathrm{pc}$ in a low absorption zone, $A_{V}=0.3$.

- The present work confirms earlier investigations that a number of cluster members are indeed variable stars: a comparison of magnitudes and colours of 30 stars in common between our CCD photometry and the photoelectric photometry of Clariá (1976) reveals that 7 stars are definitely variable and one star is probably variable, i.e. about $30 \%$ of all stars in this sample.

- The cluster has an unusual high number of stars with "peculiar colours" which are candidates for metallic line stars, probably constituting a sequence of Am to Fm. It is a known fact that peculiar A-type stars are frequently found in open clusters with ages from $10^{6}$ to $10^{8} \mathrm{yr}$. All our stars with "peculiar colours" exhibit photometric characteristics that are typical for Am stars: they lie below the $[(U-B) /(B-V)]$ intrinsic line in the two-colour diagram (Fig. 4); they mainly have absolute magnitudes in the required range as found by Abt (1979) for open cluster Am-type stars; some of these potential Am stars are variable as well.

- Kilambi (1978) noticed the presence of a gap in the stellar distribution along the cluster's MS. We demonstrated that this gap is not produced by a random process but is a real lack of stars in a given magnitude interval.

It is worth mentioning that not only open clusters show gaps but also field star distributions. Mermilliod (1976) noticed that two gaps start at the limit where the Ap and the Am stars appear, i.e. near spectral types B7 V and $\mathrm{A} 7 \mathrm{~V}$, respectively. In NGC 2571 the gap is closely connected to the position of the probable Am-type stars. Furthermore, it occupies the same location that the gap found in NGC 6531 (Forbes 1996), a young cluster of $8 \mathrm{Myr}$ only, i.e. about $40 \mathrm{Myr}$ younger than NGC 2571 . We conclude that different mechanisms of gap production are at work for clusters of different age: the gaps in the MSs of intermediate-age clusters are likely the consequence of the onset of convection in the stellar envelopes (BöhmVitense \& Canterna (1974), whereas in young clusters the gaps may be produced by the "burn-off" of ${ }^{3} \mathrm{He}$ in stars contracting to the MS (Ulrich 1971b). 
- WDs in old open clusters have been successfully searched for (e.g. von Hippel et al. 1995); but, as far as we know, for intermediate-age clusters the results were not always convincing or even negative (Anthony-Twarog 1981; Romanishin \& Angel 1980). Using the absolute magnitude of the candidate star No. 1357 as the most secure parameter, we find no contradiction in considering this star to be a cluster WD in the intermediate-age cluster NGC 2571.

A strong criterium for a WD to be a cluster member is that the sum of the evolutionary time of its progenitor, $t$ (evolution), and its cooling age, $t$ (cooling), is of the order of the cluster's age. The mass distribution of NGC 2571 (Table 5 ) gives a mean mass at the TO of $\approx 8 \mathcal{M}_{\odot}$, thus allowing us to consider star No. 1357 as the remnant of the evolution of an even more massive star, say about $9 \mathcal{M}_{\odot}$ (i.e. $1 \mathcal{M}_{\odot}$, conservatively, above the cluster TO). [We note that progenitors of WDs may have masses up to the order of $11 \mathcal{M}_{\odot}$ that had suffered strong mass loss D'Antonna \& Mazzitelli 1990; Weidemann \& Koester 1983; Weidemann 1990. We know that a WD progenitor of $9 \mathcal{M}_{\odot}$ spends about $30 \mathrm{Myr}$ to completely exhaust its nuclear helium (Schaller et al. 1992) while, on the other hand star No. 1357 as WD may be as young as $11_{-3}^{+10} \mathrm{Myr}$ (Sect. 3.6). Thus, the sum $t$ (evolution) $+t$ (cooling) is smaller than the cluster age favouring its membership. Even the less favourable case, where temperature and age of the WD candidate are derived from the $(B-V)$ index, indicates that the WD (if cluster member) is $74_{-30}^{+50} \mathrm{Myr}$ old - showing still a weak agreement between the stellar evolutionary time spent prior to the WD phase plus the cooling age of the WD with the cluster age.

- The MF of NGC 2571 was investigated in the mass range from $1 \mathcal{M}_{\odot}$ to $\approx 8 \mathcal{M}_{\odot}$. The slope of the cluster MF varies from 1.66 to 1.74 , depending on whether the most massive bin is included or not; but, on average, it is larger than the Salpeter's (1955) one. It is worth mentioning that with Scalo's (1986) relations (1.7 and 1.8) and a cluster MF's slope $x=1.74$, the expected number of stars more massive than $8.5 \mathcal{M}_{\odot}$ in NGC 2571 is 2.1 . That strongly increases the chance that the WD candidate star No. 1357 is the result of the evolution of a massive cluster member.

Acknowledgements. The authors are gateful to the referee Dr. G. Carraro for carefully reading their manuscript and for valuable comments. We also thank Dr. H. Levato for helpful discussions on peculiar stars. J.-M.W. wishes to thank ESO for granting observing time and the kind hospitality at La Silla mountain.

This article is partially based in the "Second Epoch Survey" of the southern sky that was made by the Anglo-Australian Observatory (AAO) with the UK Schmidt Telescope. Plates from this survey have been digitized and compressed by the STScI. The digitized images are copyright (c) 1993-7 by the Anglo-Australian Observatory Board.

\section{References}

Abt, H. A 1979, ApJ, 230, 485

Ahumada, J., \& Lapasset, E. 1995, A\&AS, 109, 375
Anthony-Twarog, B. J. 1981, ApJ, 245, 247

Baumgardt, H., Dettbarn, C., \& Wielen, R. 2000, A\&AS, 146, 251

Berthet, S. 1992, A\&A, 253, 451

Bergeron, P., Wesemael, F., \& Beauchamp, A. 1995, PASP, 107,1047

Böhm-Vitense, E. 1982, ApJ, 255, 191

Böhm-Vitense, E., \& Canterna, R. 1974, ApJ, 194, 629

Breger, M. 1979, PASP, 91, 5

Cannon, A. J., \& Pickering, E. C. 1918-1924, Ann. Obs. Harvard Coll. Obs. 91 to 99 and 100

Clariá, J. J. 1976, PASP, 88, 225

Cousins, A. W. J. 1978, MNRAS South Africa, 37, 62

D'Antonna, F., \& Mazzitelli, I. 1990, ARA\&A, 28, 139

Dean, J. F., Warren, P. R., \& Cousins, A. W. J. 1978, MNRAS, 183,569

de Bruijne, J. H. J., Hoogerwerf, R., \& de Zeeuw, P. T. 2000, ApJ, 544, L65

Eggen, O. J. 1976, PASP, 88, 402

ESA 1997, The Hipparcos and Tycho Catalogues (ESA Publ. Div., Noordwijk) ESA-SP-1200

Feinstein, A. 1974, AJ, 79, 11

Feinstein, A., Vázquez, R. A., \& Benvenuto, O. G. 1986, A\&A, 159,223

Forbes, D. 1996, AJ, 112, 1073

Girardi, L., Bressan, A., Bertelli, G., \& Chiosi, C. 2000, A\&A, 141,371

Golay, M. 1974, Introduction to Astronomical Photometry (Reidel Publishing Company), 81

Herbst, W., \& Miller, D. P. 1982, AJ, 87, 1478

Houk, N. 1982, Michigan Catalogue of Two-Dimensional Spectral Types for the HD Stars, vol. 3 (Dept. of Astron., Univ. of Michigan, Ann Arbor)

Kilambi, G. C. 1978, PASP, 90, 721

Landolt, A. U. 1992, AJ, 104, 340

Lindoff, U. 1968, Ark. Astron., 4, 39

Luyten, W. J., \& Herbig, G. H. 1960, Harvard Coll. Obs. Announc. Card, No. 1474

Malaroda, S. 1973, PASP, 85, 328

Mermilliod, J.-C. 1976, A\&A, 53, 289

Mermilliod, J.-C. 1981, A\&AS, 44, 467

Mermilliod, J.-C., \& Maeder, A. 1986, A\&A, 158, 45

Metanomski, A. 2000, priv. comm.

Meynet, G. J.-C., Mermilliod, \& Maeder, A. 1993, A\&AS, 98, 477

Moitinho, A. 2001, A\&A, 370, 436

Neckel, Th., \& Klare, G. 1980, A\&AS, 42, 251

Phelps, R. L., \& Janes, K. A. 1993, AJ, 106, 5

Rachford, B. J., \& Canterna, R. 2000, AJ, 119, 1296

Romanishin, W., \& Angel, J. R. P. 1980, ApJ, 235, 992

Salpeter, E. E. 1955, ApJ, 121, 161

Scalo, J. M. 1986, Fund. Cosm. Phys., 11, 1

Schaller, G., Schaerer, D., Meynet, G., \& Maeder, A. 1992, A\&AS, 96, 269

Schmidt-Kaler, Th. 1982, in Landolt-Börnstein, N. S., Group VI, vol. 2b, ed. K. Schaifer, \& H.-H. Voigt (Springer, Berlin), 1 and 449

Stetson, P. B. 1987, PASP, 99, 191

Tarrab, I. 1982, A\&A, 109, 285

Turner, D. G. 1993, A\&AS, 97, 755

Ulrich, R. K. 1971a, ApJ, 165, L95

Ulrich, R. K. 1971b, ApJ, 168, 57

Vogt, N., \& Moffat, A.F.J. 1972 A\&AS, 7, 133

von Hippel, T., Gilmore, G., \& Jones, D. H. P. 1995, MNRAS, 273, L39

Weidemann, V., \& Koester D. 1983, A\&A, 121, 77

Weidemann, V. 1990, ARA\&A, 28, 103 